\title{
Parent-child perceptions of quality of life: Implications for health intervention
}

TANIA GASPAR, PHD ${ }^{1}$

FMH/Universidade Técnica de Lisboa, CMDT/IHMT/UNL, Portugal

Margarida Gaspar de Matos, PhD

FMH/ Universidade Técnica de Lisboa, CMDT/IHMT/UNL, Portugal

JoAn Batista-Foguet, PhD

ESADE - University Ramon Llull, Barcelona, Spain

José Luís PAIS RiBeIRO, PHD

FPCE - Universidade do Porto, Portugal

ISABEL LeAL, PhD

Instituto Superior de Psicologia Aplicada - Lisboa, Portugal

\section{ABSTRACT}

The KIDSCREEN-52 is an instrument that assesses 10 dimensions of health-related quality of life (HRQoL). It was developed as a result of studies by the European KIDSCREEN Group, University of Berlin (www.kidscreen.org; see also Bisegger et al., 2005). During the Portuguese validation process, a model was developed to examine the perceptions of children and their parents on these dimensions. Structural equation modelling was used in order to estimate the fit of this model, in both cases according to gender and age. The specific aim of the present study was to examine the extent to which results differ by gender and age. An additional aim was to explore differences between the child and parent versions of the instrument, globally as well as by gender and age of the children. The results are based on a nationally representative sample of 3195 children from 5 th and 7 th grades. Data from each child were paired with data from their parents (2256 matched sets of data were generated). Most of the subscales exhibited good internal consistency in both the children's and parent's versions of KIDSCREEN-52, with values of the alpha coefficient approaching or above 80 for most scales. The exception was the subscale concerned with self-perception where the coefficient was approximately .64 for both children and parents. Subscale scores for children's and parents' versions correlated moderately strongly in the sample of matched pairs. This indicates that children and their parents view their health-related quality of life consistently,

Correspondence to: Dr Tania Gaspar, FMH /UTL - Projecto Aventura Social, Estrada da Costa, Cruz Quebrada, 1499

Lisboa Portugal. Email: taniagaspar@fmh.utl.pt

A PhD grant from FCT - SFRH/BD/22908/2005 - Portugal, Coordenação Nacional para a Infecção /HIV and

Fundação para a Ciência e Tecnologia is acknowledged. 
Tania Gaspar, Margarida Gaspar de Matos, Joan Batista- Foguet, José Luís Pais Ribeiro and Isabel Leal

although parents tend to perceive their children's quality of life as better than their children do. Analysis of variance suggested that there were small differences in scores associated with gender and age. The results confirm that the KIDSCREEN-52 questionnaire is a relevant instrument to estimate the perception of quality of life both in children and their parents. The findings that parents are not totally aware of their children's subjective health-related quality of life perceptions and that parents have different perceptions according to the gender and the age of their children, have implications for professional practice and intervention with families of school-aged children.

Keywords: Wellbeing; health-related quality of life; children; adolescents; parents

$\mathrm{H}$ ealth-related quality of life (HRQoL) in children and adolescents is a relatively recent focus of concern for health professionals. In the same way as it was described for adults by Ribeiro (1994), this concept needs to be applied to the wellbeing of children within an ecological perspective, which includes multiple levels of analysis; namely, self-perception and family-perception (Harding, 2001; Nelson, Laurendeau, \& Chamberland, 2001). Children's perceptions of their health-related quality of life are influenced by several factors such as gender, age, personal and familial characteristics, and socio economic status (Bronfenbrenner, 1986; Caldera \& Hart, 2004; Kazdin \& Whitley, 2003). Studies focusing on children's subjective wellbeing include interactions between demographics (e.g., age, gender and socioeconomic status), interpersonal characteristics (self-perception, feelings, general mood) and perceptions of wellbeing and happiness. (Matos, 2005; Mccullough, Huebner, \& Laughlin, 2000; Plancherel, Bolognini, \& Halfon, 1998).

In a review of research that compared selfreports of children and proxy reports of parents about health-related quality of life (Eiser \& Morse, 2001), the most common analytical strategies have included Pearson product-moment correlation coefficients, $k$-statistics, comparison of group means, and interclass correlation coefficients (ICC) (Tamim, McCusker, \& Dendukuri, 2002). The examination of Pearson correlation and ICC results provide useful evidence to evaluate the degree of agreement between children's and parents' perceptions (Eiser \& Morse, 2001; Marshall, Hays, \& Nicholas, 1994). According to
Eiser and Morse (2001), the reports of parents and children show real differences, rather than poor measurement quality of the instrument.

Parents and children can also differ from each other depending on the instrument used (Sung et al., 2004). Several studies have indicated that the level of agreement is dependent upon the health domain being examined; some parents may have limited knowledge of their children's health-related quality of life, particularly the impact on social and emotional wellbeing (Jokovic, Locker, \& Guyatt, 2004). On the other hand, according to Chang and Yeh (2005), parents' reports of quality of life can be used as a proxy report for children who are younger than 12 years of age, though not so effectively for adolescents.

The Bisegger et al. and KIDSCREEN Group (2005) found clear gender and age differences in children and adolescents, when using the KIDSCREEN-52 measuring instrument. This instrument was developed within the European project 'Screening and Promotion for Health-Related Quality of Life in Children and Adolescents - A European Public Health Perspective' (European Commission, with the aim of building a standardized instrument to estimate the subjective quality of life in children and adolescents and their parents. Over 3 years (2001-2004), 13 countries co-ordinated by the German team (Ravens-Sieberer et al. \& European KIDSCREEN Group, 2001) developed and evaluated this instrument, presenting a version for Children and a version for Parents, which can be used with children from 8-18 years of age, and their parents. Portugal joined this group in 2004 in order to provide a Portuguese version. 
The aim of the present study was to evaluate the psychometric properties of the Portuguese version of the KIDSCREEN-52 instrument (Gaspar \& Matos, 2008), as well as analysing results by gender, age and differences between the children's and parents' versions. It had been translated and piloted for the Portuguese population in 2005 , using a strict translation/adaptation protocol, as requested by the European Group (Gaspar \& Matos, 2008; Gaspar, Matos, Ribeiro, \& Leal, 2005; 2006; Gaspar, Matos, Ribeiro, Leal \& Ferreira, 2009a; 2009b; Gaspar, Matos, Ribeiro, Leal, Erhart, \& Ravens-Sieberer, 2010).

\section{METHOD}

\section{Measuring instrument}

KIDSCREEN-52 can be used to measure, monitor, and evaluate subjective health-related quality of life in children and adolescent populations. It can be used in schools, hospitals, and in the research field - in areas such as public health and health psychology, and epidemiology.). KIDSCREEN-52 is a 52 -item self-report questionnaire, reporting on the 'past week' and requires about 15 minutes to complete. It includes 10 dimensions, describing healthrelated quality of life (Ravens-Sieberer et al. \& European KIDSCREEN Group, 2001): Health \& Physical Activity; General Mood/Emotions; Feelings; Self-Perception; Free Time\& Autonomy; Family \& Family Context; Friends \& Social context; School \&Learning; Money Matters; and Bullying (Ravens-Sieberer et al. \& European KIDSCREEN Group, 2001). To score the instrument, 14 items need to be reverse coded in order to have all items formulated positively (i.e.,a higher score reflects a higher HRQoL perception). The score range for each dimension is 0 to 100 . The statistical software package SPSS 15 was used.

\section{Sampling and procedure}

Sampling methods were derived from the international study 'Health Behaviour School Aged-Children' (HBSC/WHO). The HBSC/ WHO study's sample was enlarged, and two extra random classes (5th grade and 7th grade) were selected in each of the national randomly selected schools. Schools were stratified by National Educational Regions (five in the whole country), following HBSC/ WHO. (For further details about sampling procedures, see Currie, Samdal, Boyce, \& Smith, 2001; Matos et al., 2006, and www.hbsc.org). The present study is thus a cross-sectional national study, representative of Portuguese public schools (except Portuguese islands), and provides a random national representative sample of 5 th and 7 th grade pupils, matched with their parents.

Questionnaires for children and parents were numbered twice so that they could be paired afterwards without violating anonymity. All ethical issues and legal authorizations, as well as consent from parents and schools were obtained. KIDSCREEN-52 questionnaires were administered by teachers in a classroom setting. Questionnaires were anonymous and answered voluntarily.

The study involved 92 schools and 162 classes. A sample of 3195 children (81\% response rate) from $5^{\text {th }}$ grade $(48.8 \%)$ and 7 th grade $(51.2 \%)$, mean age 11.8 years old; $S D$ 1.46; ranging from $10-16(41.1 \%$ between 10 and 11 years of age and $58.9 \% 12$ years or older); there were $49.2 \%$ boys and $50.8 \%$ girls. Children were matched with the questionnaires from their parents if available. A total of 2256 parents filled out the questionnaires and could be matched with their children. Mostly mothers filled the questionnaire (97.8\%). The majority of pupils came from a low or very low socio-economic status $(62.2 \%)$, and $3.3 \%$ did not have Portuguese nationality. A single database was built that contained the responses of both children and their parents. At this point, children whose parents did not complete the questionnaire were left out.

\section{RESULTS}

The KIDSCREEN-52 instrument showed good internal consistency for almost all of the 10 subscales. Tables 1 and 2 show, marked in bold, the mean values with statistical significance (higher mean in bold). In the children's version, the low- 
est value was self-perception $(\alpha=.60)$ and the highest money matters $(\alpha=.88)$ (see Table 1$)$. The internal consistency of HRQoL Total (all dimensions together) is good at $\alpha=.87$. Similar results occurred with the Parents' version: the lowest value of internal consistency reported was self-perception $(\alpha=.64)$ and the highest money matters $(\alpha=.87)$ (see Table 2$)$. The internal consistency of HRQoL Total is good at $\alpha=.87$.

In the children's version of the instrument almost all subscales presented values of internal consistency higher than .80 , except the dimensions self-perception $(\alpha=.60)$, health $\&$ physical activity $(\alpha=.77)$, and bullying $(\alpha=.75)$. In the parents version of the instrument, all subscales presented values of internal consistency higher than .80 , except the dimensions of self-perception $(\alpha=.64)$.

All HRQoL dimensions and the Total scale were statistically significantly correlated (Pearson r), either in the children's or in the parents' version. In the children's version, the highest correlations occurred between feelings and free time $\&$ autonomy and other KIDSCREEN-52 dimensions: feelings and general mood/emotions $(r=.63 ; p<.01)$, feelings and family \& family context $(r=.56 ; p<$ $.01)$, feelings and free time $\&$ autonomy ' $(r=.56$;

TABLE 1: SCALE DESCRIPTIVE ANALYSIS AND INTERNAL CONSISTENCY OF KIDSCREEN-52 DIMENSIONS IN PORTUGAL - CHILDREN'S VERSION

\begin{tabular}{|c|c|c|c|c|c|c|c|}
\hline Dimensions & $\begin{array}{l}\text { No. } \\
\text { items }\end{array}$ & $N$ & $M$ & $M \%^{*}$ & $S D$ & $\begin{array}{c}\text { Cronbach } \\
\alpha\end{array}$ & $\begin{array}{l}\text { Cronbach } \\
\alpha \text { range }^{\star *}\end{array}$ \\
\hline 1. Health \& physical activity & 5 & 3065 & $19.34(0-25)$ & 71.68 & 17.58 & .77 & $.75-.86$ \\
\hline 2. Feelings & 6 & 3111 & $25.22(0-30)$ & 80.08 & 19.88 & .84 & $.85-.91$ \\
\hline 3. General moods \& emotions & 7 & 3019 & $28.52(0-35)$ & 76.86 & 19.19 & .86 & $.80-.89$ \\
\hline 4. Self-perception & 5 & 3085 & $19.72(0-25)$ & 73.61 & 18.22 & .60 & $.71-.84$ \\
\hline 5. Free time \& autonomy & 5 & 3082 & $20,29(0-25)$ & 76.46 & 20.95 & .81 & $.79-.86$ \\
\hline 6. Family \& family context & 6 & 3092 & $25.22(0-30)$ & 80.10 & 19.84 & .84 & $.85-.90$ \\
\hline 7. Money matters & 3 & 3100 & $11.91(0-15)$ & 74.21 & 27.15 & .88 & $.82-.91$ \\
\hline 8. Friends \& social context & 6 & 3058 & $24,49(0-30)$ & 77.04 & 19.73 & .84 & $.81-.87$ \\
\hline 9. School \& learning & 6 & 3100 & $22.71(0-30)$ & 69.64 & 20.11 & .84 & $.81-.88$ \\
\hline 10. Bullying & 3 & 3144 & $12.60(0-15)$ & $79 . .8$ & 22.05 & .75 & $.61-.83$ \\
\hline
\end{tabular}

* Sum score transformed into values between 0-100.

*^ Range across countries - European KIDSCREEN Group (Ravens-Sieberer \& European KIDSCREEN Group, 2005).

TABLE 2: SCALE DESCRIPTIVE ANALYSIS AND INTERNAL CONSISTENCY OF KIDSCREEN-52 DIMENSIONS IN PORTUGAL - PARENTS' VERSION

\begin{tabular}{lcccccc} 
& No. & \multicolumn{2}{c}{} & \multicolumn{2}{c}{ Cronbach } \\
items & $\boldsymbol{N}$ & $\boldsymbol{M}$ & $\boldsymbol{M}$ \% $^{*}$ & SD & $\boldsymbol{\alpha}$ \\
\hline 1. Health \& physical activity & 5 & 2182 & $19.36(0-25)$ & 71.82 & 18.03 & .82 \\
2. Feelings & 6 & 2115 & $25.43(0-30)$ & 80.96 & 14.68 & .85 \\
3. General moods \& emotions & 7 & 2131 & $29.75(0-35)$ & 81.25 & 15.87 & .85 \\
4. Self-perception & 5 & 2147 & $20.49(0-25)$ & 77.46 & 16.04 & .64 \\
5. Free time \& autonomy & 5 & 2137 & $20.84(0-25)$ & 79.21 & 17.65 & .81 \\
6. Family \& family context & 6 & 2106 & $26.08(0-30)$ & 83.66 & 14.89 & .80 \\
7. Money matters & 3 & 2140 & $11.53(0-15)$ & 71.10 & 25.27 & .87 \\
8. Friends \& social context & 6 & 2061 & $23.05(0-30)$ & $7 ., 05$ & 18.61 & .86 \\
9. School \& learning & 6 & 2111 & $23.58(0-30)$ & 73.24 & 16.81 & .85 \\
10. Bullying & 3 & 2117 & $12.68(0-15)$ & 80.70 & 20.59 & .83 \\
\hline
\end{tabular}

* Sum score transformed into values between 0-100. 
$p<.01)$, feelings and health \& physical activity $(r$ $=.54 ; p<.01)$ and also free time $\&$ autonomy and friends $\&$ social context $(r=.57 ; p<.01)$ and free time $\&$ autonomy and family $\&$ family context $(r$ $=.53 ; p<.01)$. The HRQoL Total scale showed the highest correlation with the feelings dimension and the lowest correlation with the school \& learning dimension (see Table 3).

In the parents' version, the highest correlations occurred between feelings and general mood/ emotions $(\mathrm{r}=.58 ; \mathrm{p}<.01)$, feelings and family $\&$ family context $(r=.56 ; p<.01)$, feelings and health \& physical activity $(r=.54 ; p<.01)$, free time $\&$ autonomy and friends \& social context $(r$ $=.53 ; p<.01)$, and family \& family context and general mood/emotions $(r=.52 ; p<.01)$. As with the children's results, the hrqol total scale showed the higher correlation with the feelings dimension and the lower correlation with the school \& learning dimension (see Table 4).

Gender and age differences concerning children's perceptions of health-related quality of life were identified using analysis of variance. Parents' answers were paired and compared with those of their children ( $t$ test for repeated measures). Gender and age differences were also identified,

\section{Table 3: Pearson correlation between KidsCReen-52 dimensions in Portugal - Children's} VERSION

\begin{tabular}{|c|c|c|c|c|c|c|c|c|c|c|}
\hline KIDSCREEN-52 dimension & 1 & 2 & 3 & 4 & 5 & 6 & 7 & 8 & 9 & Total \\
\hline 1. Health \& physical activity & - & & & & & & & & & \\
\hline 2. Feelings & $.54^{\star \star}$ & - & & & & & & & & \\
\hline 3. General moods \& emotions & $.36^{\star \star}$ & $.63^{* *}$ & - & & & & & & & \\
\hline 4. Self-perception & $.29^{\star \star}$ & $.44^{\star \star}$ & $.50 \star \star$ & - & & & & & & \\
\hline 5. Free time \& autonomy & $.43^{\star \star}$ & $.56^{\star \star}$ & $.48^{\star \star}$ & $.39 \star \star$ & - & & & & & \\
\hline 6. Family \& family context & $.33^{\star \star}$ & $.56^{\star \star}$ & $.50^{\star \star}$ & $.43^{\star \star}$ & $.53^{* *}$ & $\longrightarrow$ & & & & \\
\hline 7. Money matters & $.28^{\star *}$ & $.35^{\star \star}$ & $.32^{\star \star}$ & $.27^{\star \star}$ & $.44^{\star \star}$ & $.45^{\star \star}$ & 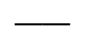 & & & \\
\hline 8. Friends \& social context & $.39 \star \star$ &. $.49^{\star \star}$ & $.42^{\star \star}$ & $.33^{\star \star}$ & $.57^{\star \star}$ & $.44^{\star \star}$ & $.48^{\star \star}$ & - & & \\
\hline 9. School \& learning & $.27^{\star \star}$ & $.48^{\star \star}$ & $.43^{\star \star}$ & $.34^{\star \star}$ & $.37^{\star \star}$ & $.44^{\star \star}$ & $.29 \star \star$ & $.37^{\star \star}$ & $\underline{.}$ & \\
\hline 10. Bullying & $.22^{\star \star}$ & $.27^{\star \star}$ & $.41^{\star \star}$ & $.33^{\star \star}$ & $.24^{\star \star}$ & $.24^{\star \star}$ & $.30^{\star \star}$ & $.33^{\star \star}$ & $.14^{\star \star}$ & \\
\hline $\mathrm{RO}$ & $.60^{\star *}$ & $.78^{\star \star}$ & $.75^{\star \star}$ & $.64^{\star \star}$ & $.74^{\star *}$ & $.74^{\star \star}$ & $.73^{\star \star}$ & $.62^{\star \star}$ & $.55^{\star *}$ & $.66 * *$ \\
\hline
\end{tabular}

** $p<.01$, two-tailed

Table 4: Pearson correlation between KidsCreen-52 dimensions in Portugal - Parents' VERSION

\begin{tabular}{|c|c|c|c|c|c|c|c|c|c|c|}
\hline KIDSCREEN-52 dimension & 1 & 2 & 3 & 4 & 5 & 6 & 7 & 8 & 9 & Total \\
\hline 1. Health \& physical activity & - & & & & & & & & & \\
\hline 2. Feelings & $.53^{\star \star}$ & - & & & & & & & & \\
\hline 3. General moods \& emotions & $.31 \star \star$ & $.57^{\star \star}$ & - & & & & & & & \\
\hline 4. Self-perception & $.24^{\star \star}$ & $.41^{\star \star}$ & $.44^{\star \star}$ & - & & & & & & \\
\hline 5. Free time \& autonomy & $.34^{\star \star}$ & $.49^{\star *}$ & $.37^{\star \star}$ & $.31^{\star \star}$ & - & & & & & \\
\hline 6. Family \& family context & $.31 \star \star$ & $.57^{\star \star}$ & $.52^{\star \star}$ & $.41^{\star \star}$ & $.47^{\star \star}$ & - & & & & \\
\hline 7. Money matters & $.24^{\star \star}$ & $.28^{\star \star}$ & $.28^{\star \star}$ & $.21^{\star \star}$ & .34 * & $.34^{\star \star}$ & - & & & \\
\hline 8. Friends \& social context & $.37^{\star \star}$ & $.43^{\star \star}$ & $.34^{\star \star}$ & $.21^{\star \star}$ & $.51^{\star \star}$ & $.38^{\star *}$ & $.42^{\star \star}$ & - & & \\
\hline 9. School \& learning & $.28^{\star \star}$ & $.46^{\star \star}$ & $.43^{\star \star}$ & $.34^{\star \star}$ & $.34 * \star$ & $.49^{\star \star}$ & $.29 * \star$ & $.35^{\star \star}$ & - & \\
\hline 10. Bullying & $.19^{\star \star}$ & $.25^{\star \star}$ & $.37^{\star \star}$ & $.27^{\star \star}$ & $.20 \star \star$ & $.22^{\star \star}$ & $.26^{\star \star}$ & $.30^{\star \star}$ & $.23^{\star \star}$ & 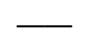 \\
\hline $\mathrm{HRQ}$ & $.58^{\star \star}$ & $.74^{\star \star}$ & $.71^{\star *}$ & $.58^{\star \star}$ & $.68^{\star *}$ & $.70^{\star \star *}$ & $.68^{* *}$ & $.64^{\star \star}$ & $.53^{\star \star}$ & $.62^{\star *}$ \\
\hline
\end{tabular}

${ }^{\star *} p<.01$, two-tailed. 
concerning parents' perceptions of their children's health-related quality of life. Structural equation modelling was used to estimate the fit of a model of children's and parents' perceptions on HRQoL according to gender and age. Both models were adjusted. Girls' perceptions of quality of life was poorer than boys on all dimensions at the statistically significant level of $p<.001$, with the exception of friends \& social context and money matters, and of school \& learning, where girls had a more positive perception than boys. The effect size analysis highlighted differences between boys and girls in self-perception, free time $\&$ autono- my, school \& learning and especially on the health $\&$ physical activity dimension (see Table 5).

The older group of students (those in the $7^{\text {th }}$ grade) presented a poorer perception of quality of life than the younger group on all measures except money matters, where there were no statistically significant differences, and bullying where the older group showed better results (low bullying). The effect size analysis revealed differences between children and adolescents in feelings, general mood/emotions, free time \& autonomy, family \& family context and especially on the self-perception and school \& learning dimensions (see Table 6).

TABle 5: Means and Standard deviations and ANOVAs - Children's HRQol - Gender COMPARISONS - CHILDREN'S VERSION $(n=3195)$

\begin{tabular}{|c|c|c|c|c|c|c|}
\hline \multirow[b]{2}{*}{ Dimensions } & \multicolumn{2}{|c|}{ Boys } & \multicolumn{2}{|c|}{ Girls } & \multirow[b]{2}{*}{$F$} & \multirow[b]{2}{*}{ Effect size } \\
\hline & $M$ & $S D$ & $M$ & $S D$ & & \\
\hline Health \& physical activity & 75.64 & 1.69 & 67.93 & 17.59 & $154.49^{\star \star \star}$ & .44 \\
\hline Feelings & 81.14 & 16.45 & 79.06 & 17.22 & $11.89 * \star \star$ & .12 \\
\hline General mood/emotions & 78.52 & 18.66 & 75.29 & 19.57 & $21.60^{\star \star *}$ & .16 \\
\hline Self-perception & 76.07 & 17.14 & 71.25 & 18.90 & $54.94^{\star \star \star}$ & .26 \\
\hline Free time \& autonomy & 78.75 & 20.39 & 74.21 & 21.26 & $35.92^{\star \star \star}$ & .21 \\
\hline Family \& family context & 81.70 & 18.61 & 78.55 & 20.84 & $19.56^{\star \star \star}$ & .16 \\
\hline Money matters & 74.13 & 27.44 & 74.29 & 26.87 & (n.s.) & (n.s.) \\
\hline Friends \& social context & 77.15 & 19.77 & 76.93 & 19.70 & (n.s.) & (n.s.) \\
\hline School \& learning & 67.00 & 21.63 & 72.17 & 18.18 & $51.97^{\star \star \star}$ & .26 \\
\hline Bullying & 81.11 & 21.54 & 78.89 & 22.49 & $8.00^{\star \star *}$ & .10 \\
\hline
\end{tabular}

${ }^{* \star *} p \leq .001$

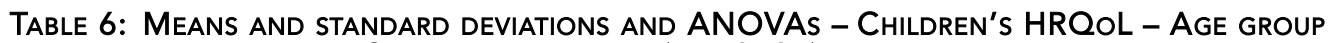
COMPARISONS - CHILDREN'S VERSIONS $(n=3195)$

\begin{tabular}{|c|c|c|c|c|c|c|}
\hline \multirow[b]{2}{*}{ Dimensions } & \multicolumn{2}{|c|}{ Age: $10-11$ years } & \multicolumn{2}{|c|}{ Age: 12 years or older } & \multirow[b]{2}{*}{$F$} & \multirow[b]{2}{*}{ Effect size } \\
\hline & $M$ & $S D$ & $M$ & $S D$ & & \\
\hline Health \& physical activity & 72.75 & 17.17 & 70.94 & 17.83 & $7.84^{\star \star}$ & .10 \\
\hline Feelings & 83.01 & 15.09 & 78.02 & 17.74 & $67.35^{\star \star \star}$ & .30 \\
\hline General mood/emotions & 80.39 & 17.58 & 74.40 & 19.88 & $72.77^{\star \star \star}$ & .31 \\
\hline Self-perception & 78.66 & 17.03 & 70.13 & 18.19 & $172.66^{\star \star \star}$ & .47 \\
\hline Free time \& autonomy & 79.43 & 19.46 & 74.41 & 21.70 & $43.22^{\star \star \star}$ & .24 \\
\hline Family \& family context & 83.75 & 17.08 & 77.55 & 21.19 & $74.65^{\star \star \star}$ & .31 \\
\hline Money matters & 74.47 & 27.22 & 74.03 & 27.10 & (n.s.) & (n.s.) \\
\hline Friends \& social context & 78.40 & 18.78 & 76.09 & 20.32 & $10.16^{\star \star \star}$ & .11 \\
\hline School \& learning & 77.22 & 17.52 & 64.27 & 20.10 & $346.64^{\star \star \star}$ & .69 \\
\hline Bullying & 78.23 & 22.13 & 81.21 & 21.92 & $14.00^{\star \star *}$ & .13 \\
\hline
\end{tabular}

${ }^{* \star} p \leq .01 ;{ }^{* \star *} p \leq .001$ 
Parents tended to perceive their children's quality of life as better than their children perceived it to be, with the exception of the health \& physical activity, feelings and bullying dimensions, where there were no statistically significant differences; they also perceived money matters and friends \& social context dimensions in a more negative way than their children. The effect size analysis highlighted the differences between parents and their children's perceptions on general moods /emotions, self-perception, and especially friends \& social context dimensions (see Table 7). However, the correlation on the
HRQoL Total between children and parents is very low and not statistically significant.

Parents tended to perceive their daughters and sons as having better quality of life than did their children on the dimensions of school \& learning, bullying and money matters (for girls); and health \& physical activity, self-perception, and free time $\&$ autonomy (for boys). The effect size analysis highlighted these differences, especially on the health \& physical activity, self-perception and school \& learning dimensions (see Table 8).

Parents tended to perceive their younger children ( $5^{\text {th }}$ grade) as having better quality of life

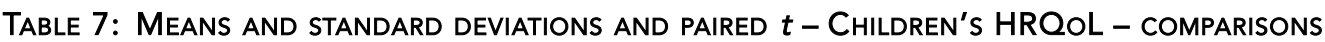
BETWEEN PARENTS AND CHILDREN'S VERSIONS $(n=4460)$

\begin{tabular}{|c|c|c|c|c|c|c|c|c|}
\hline \multirow[b]{2}{*}{ Dimensions } & \multicolumn{2}{|c|}{ Children } & \multicolumn{2}{|c|}{ Parents } & \multirow[b]{2}{*}{ Paired $t$} & \multirow[b]{2}{*}{ Effect size } & \multirow[b]{2}{*}{$r$} & \multirow[b]{2}{*}{$p$} \\
\hline & $M$ & $S D$ & $M$ & $S D$ & & & & \\
\hline Health \& physical activity & 71.67 & 17.53 & 71.92 & 18.02 & (n.s.) & (n.s.) & .02 & .31 \\
\hline Feelings & 80.90 & 16.22 & 80.94 & 14.71 & (n.s.) & (n.s.) & .01 & .82 \\
\hline General mood/emotions & 78.16 & 18.37 & 81.23 & 15.90 & $-5.512^{\star \star \star}$ & .17 & .01 & .53 \\
\hline Self-perception & 74.35 & 18.15 & 77.48 & 16.06 & $-5.718^{\star \star \star}$ & .17 & -.03 & .12 \\
\hline Free time \& autonomy & 77.22 & 20.63 & 79.28 & 17.65 & $-3.713^{\star \star \star}$ & .11 & -.01 & .60 \\
\hline Family \& family context & 80.97 & 19.20 & 83.66 & 14.93 & $-4.811^{\star \star \star}$ & .14 & -.04 & .09 \\
\hline Money matters & 74.56 & 26.65 & 71.24 & 25.22 & $4.357^{\star \star \star}$ & .13 & .01 & .78 \\
\hline Friends \& social context & 77.58 & 19.27 & 71.08 & 18.58 & $10.717^{\star \star \star}$ & .32 & -.01 & .88 \\
\hline School \& learning & 71.91 & 19.00 & 73.24 & 16.84 & $-2.134^{\star}$ & .06 & -.03 & .25 \\
\hline Bullying & 80.01 & 21.47 & 80.63 & 20.64 & (n.s.) & (n.s.) & -.01 & .90 \\
\hline
\end{tabular}

${ }^{\star} p<.05 ;{ }^{* \star \star} p \leq .001$

TABle 8: MeANS AND STANDARd DeVIATIONS AND ANOVAs - ChILdREN's HRQOL - Gender COMPARISONS - PARENTS' VERSION $(n=2256)$

\begin{tabular}{|c|c|c|c|c|c|c|}
\hline \multirow[b]{2}{*}{ Dimensions } & \multicolumn{2}{|c|}{ Boys } & \multicolumn{2}{|c|}{ Girls } & \multirow[b]{2}{*}{$F$} & \multirow[b]{2}{*}{ Effect size } \\
\hline & $M$ & $S D$ & $M$ & $S D$ & & \\
\hline Health \& physical activity & 74.24 & 17.53 & 69.78 & 18.20 & $33.56^{\star \star *}$ & .24 \\
\hline Feelings & 81.44 & 14.40 & 80.54 & 14.91 & (n.s.) & (n.s.) \\
\hline General mood/emotions & 80.84 & 16.11 & 81.59 & 15.67 & (n.s.) & (n.s.) \\
\hline Self-perception & 79.60 & 14.95 & 75.69 & 16.69 & $32.83^{\star \star \star}$ & .24 \\
\hline Free time \& autonomy & 79.96 & 17.30 & 78.58 & 17.93 & $3.21^{\star}$ & .08 \\
\hline Family \& family context & 84.21 & 14.35 & 83.20 & 15.33 & (n.s.) & (n.s.) \\
\hline Money matters & 68.93 & 25.90 & 72.94 & 24.59 & $13.60 * \star \star$ & .16 \\
\hline Friends \& social context & 70.36 & 18.69 & 71.61 & 18.53 & (n.s.) & (n.s.) \\
\hline School \& learning & 70.95 & 17.62 & 75.22 & 15.83 & $34.32^{\star \star \star}$ & .25 \\
\hline Bullying & 79.81 & 20.68 & 81.46 & 20.50 & $3.60^{\star}$ & .08 \\
\hline
\end{tabular}

${ }^{*} p<.01 ;{ }^{* * *} p \leq .001$ 
than the older children on all the dimensions, except for friends \& social context, where there was no statistically significant difference, and for bullying, where parents perceived a better situation for the older ( $7^{\text {th }}$ grade) group. The effect size analysis revealed marked differences in parents' perceptions of the children's quality of life on feelings, general mood/emotions, self-perception, family \& family context, and especially on the school \& learning dimensions (see Table 9).

Effect size: Despite the statistically significant differences between the analysed groups, as shown in Tables 5-9, it should be noted that the effect sizes are, in general, small (i.e. < .50) and, therefore, unremarkable (Cohen, 1988).

Regression analysis: A regression model, using the HRQoL Total score (children's version) as the dependent variable and age and gender as predictors, showed that age explained $3.2 \%$ of the variance $(\beta=-.18, p<.0001)$ and gender explained $1.6 \%$ of the variance $(\beta=.13, p<.0001)$. This children's Total score was statistically significantly predicted (in all cases at the $p<.0001$ level) by all 10 dimensions ( $\beta$ ranging between .12 on the psychological wellbeing factor and .20 on the financial resources factor). A regression model, using the HRQoL Total score (parents' version) as a dependent variable and age and gender as predictors, showed that age explained $0.3 \%$ of the vari- ance $(\beta=-.06, p<.05)$ and gender explained $0.2 \%$ of the variance $(\beta=.06, p<.05)$. This parents' Total score was significantly predicted (in all cases at the $p<.001$ level) by all dimensions ( $\beta$ ranging between .13 on the psychological wellbeing factor and .22 on the financial resources factor).

\section{DISCUSSION}

The KIDSCREEN-52 questionnaire is a relevant and promising instrument to use to estimate the perception of quality of life both in children and their parents in the context of the Portuguese language and culture (Gaspar \& Matos, 2008; Gaspar et al., 2005, 2006, 2009a, 2009b, 2010), in the same way as it has been used in other countries (Bisegger et al., 2005; Ravens-Sieberer et al., 2005; Rueden et al., 2006). The Portuguese versions (Children's and Parents' versions) of the instrument show good psychometric properties and good internal consistency. The Self-Perception dimension was the only dimension with rather low internal consistency. An Exploratory Factor Analysis (EFA) carried out previously during the national validation process (Gaspar \& Matos, 2008) provided no evidence of a better model than the one used in the KIDSCREEN52, except for the third item on the self-perception domain - Have you been happy with the way you are? - which the EFA loaded onto the psycho-

Table 9: Means and standard deviations and ANOVAs - Children's HRQol - Age Group COMPARISONS - PARENTS' VERSION $(n=2256)$

\begin{tabular}{|c|c|c|c|c|c|c|}
\hline \multirow[b]{2}{*}{ Dimensions } & \multicolumn{2}{|c|}{ Age: $10-11$ years } & \multicolumn{2}{|c|}{ Age: 12 years or older } & \multirow[b]{2}{*}{$F$} & \multirow[b]{2}{*}{ Effect size } \\
\hline & $M$ & $S D$ & $M$ & $S D$ & & \\
\hline Health \& physical activity & 73.21 & 17.75 & 70.51 & 18.14 & $12.31 * \star \star$ & .15 \\
\hline Feelings & 82.43 & 13.79 & 79.49 & 15.34 & $21.32^{\star \star \star}$ & .20 \\
\hline General mood/emotions & 83.34 & 14.02 & 79.49 & 16.96 & $32.09^{\star \star \star}$ & .24 \\
\hline Self-perception & 80.25 & 15.18 & 74.87 & 16.35 & $61.74^{\star \star \star}$ & .33 \\
\hline Free time \& autonomy & 80.01 & 16.90 & 78.40 & 18.33 & $4.39 \star \star$ & .09 \\
\hline Family \& family context & 85.74 & 13.58 & 81.75 & 15.72 & $38.23^{\star \star \star}$ & .26 \\
\hline Money matters & 73.15 & 24.24 & 69.11 & 26.06 & $13.63^{\star \star \star}$ & .16 \\
\hline Friends \& social context & 71.21 & 17.67 & 70.83 & 19.43 & (n.s.) & (n.s.) \\
\hline School \& learning & 76.91 & 15.54 & 69.79 & 17.16 & 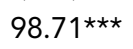 & 0.42 \\
\hline Bullying & 78.83 & 20.92 & 82.45 & 20.10 & $16.40^{\star \star \star}$ & 0.17 \\
\hline
\end{tabular}

${ }^{\star} p<.05 ;{ }^{\star \star} p \leq .01 ;{ }^{\star \star \star} p \leq .001$ 
logical wellbeing factor. This result could be related to a 'culturally specific' translation issue, perhaps because the meaning of the word ' $h a p p y$ ' in the Portuguese language is better related to the psychological wellbeing domain. During the same validation process, the analysis of the total population data confirmed 10 factors (Eigenvalues $>$ 1.0 ), in either children's or parents' versions (Gaspar \& Matos, 2008).

In order to evaluate the impact of age and gender on HRQoL scores, regression models were carried out. Age and gender differences were statistically significant for almost all the dimensions studied, but the effect sizes revealed that those differences were not very large. According to the literature, girls and adolescents express poorer HRQoL scores than boys and children, respectively (Bisegger et al., 2005; Gaspar et al., 2005; 2006; 2009a; 2009b; 2010), a finding confirmed in the present study through the good fit achieved (SEM), not only on the children's, but also on their parents' perceptions. It is noteworthy that Bisegger et al.(2005) reported girls' perception of health-related quality of life to be poorer than that of boys on all 10 dimensions assessed, except friends, school \& learning and bullying; adolescents presented a poorer perception of health-related quality of life than the younger children on all dimensions except money matters and bullying.

In the children's version, the highest correlations occurred between feelings, free time \& autonomy and other dimensions. According to the children's perceptions, feelings are related to family, friends, general mood/emotions, free time $\&$ autonomy and health \& physical activity. Free time $\&$ autonomy is related to both family and friends. these finding support the assumptions that free time \& autonomy is an important feature in children's lives, and that both parents and friends are important to provide emotional, personal and social support (Matos, 2005).

In the parents' version, the highest correlations occurred between feelings and other dimensions. According to parents' perceptions, feelings are related to general mood/emotions, family, and health \& physical activity; family is more highly related to general mood/emotions, and friends to free time $\&$ autonomy. This is an interesting bias, with parents valuing family as more highly associated with general mood/ emotional states, while friends are seen to be associated with free time $\&$ autonomy.

Several differences can be found in healthrelated quality of life perceptions as reported by parents and children. It is assumed that this reflects real differences in perception rather than poor measurement quality of the instrument (Eiser \& Morse, 2001). Clear gender and age differences can be found in HRQoL scores, as reported by the children in this study. The perception of girls in both age groups of quality of life was found to be poorer than boys' perceptions on all dimensions except friends \& social context and money matters (where there were no statistically significant differences), and school \& learning (where girls achieved better results). Other KIDSCREEN studies have shown similar results; girls' perception of health-related quality of life is poorer than boys on all dimensions except friends $\&$ social context, school \& learning and bullying (Ravens-Sieberer et al., 2005). This also confirms the findings from the literature about girls' poor perception of happiness and stronger investment in school (Matos et al., 2006).

The older group of children in the study reported a poorer perception of quality of life compared with the younger group on all measures except money matters, where there were no statistically significant differences; and bullying, where the older children reported more positive perceptions. Similar results can be found in RavensSieberer et al. (2005), in which the authors found that the older group of children presented a poorer perception of health-related quality of life on all measures except money matters and bullying. Results from both studies also confirm previous literature, namely, that bullying as a concern decreases as children progress through adolescence (Matos, 2005; Matos et al., 2006).

Parents tended to perceive their children's quality of life as better than their children did, 
except in the areas of health \& physical activity, feelings and bullying, where there were no statistically significant differences; and parents considered money matters and friends dimensions more negatively than did their own children. The correlations between the children and parents' versions of KIDSCREEN-52 are not statistically significant. In general, the versions can be considered to be rather independent of each other. Proxy results (parents' version) cannot thus be a direct substitute for results from the children's version of the instrument. It is important and relevant to know and understand both perspectives of perception of quality of life. Furthermore, parents tended to perceive their daughters as having better quality of life in the dimensions of school $\&$ learning, bullying and money matters, and to perceive their sons as having a better quality of life in the health \& physical activity, self-perception, and free time $\&$ autonomy dimensions. Parents tended to perceive their younger children as having better quality of life on all the dimensions, except free time \& autonomy and friends \& social context, where there were no statistically significant differences; however, on the Bullying dimension, parents reported less Bullying concern in the older group ( $7^{\text {th }}$ grade) than in the younger group.

Several studies have indicated that the level of agreement between parent and child perceptions is dependent upon the health domain being examined. Some parents may have limited knowledge about their children's health-related quality of life perceptions, particularly about the impact on social and emotional wellbeing (Jokovic et al., 2004). The present study has confirmed that parents' proxy-reporting of quality of life is useful as a substitute report for children who are younger than 12 years of age, but not so effective for adolescents (Chang \& Yeh, 2005). Our results present an interesting bias in parents' evaluation of their children's quality of life, suggesting that children over-evaluate (or parents under-evaluate) the economic aspect of their lives, and the impact of friends.
In general, parents estimated their daughters as having a better experience of schooling and less involvement in bullying, and their sons as having a better health and physical activity, a better selfperception and better free time/autonomy. Present findings agree with the general literature on gender differences and also with the general gender differences in beliefs, which suggest that both children and their families are prone to perceive gender differences. They also corroborated previous research either in the area of health-related quality of life or in the area of developmental psychology (Caldera \& Hart, 2004; Harding, 2001; Kazdin $\&$ Whitley, 2003; Matos et al., 2006; Nelson et al., 2001). The fact that parents are not totally aware of their children's subjective health-related quality of life perceptions and that parents perceive a difference according to the gender and the age of their children has implications for intervention with families of school-aged children.

\section{CONCLUSION}

The present report on the national validation of KIDSCREEN-52 in Portugal has found that children and parents are able to estimate a latent measure of health-related quality of life based on 10 dimensions contained in the KIDSCREEN52 questionnaire. It appears to be a relevant instrument to estimate the perception of quality of life both in children and their parents, allowing for the identifying of gender and age differences. Bearing in mind the perceptions that parents have about their children's health-related quality of life, and how well they match or do not match their children's own perceptions, this study contributes to improving the evaluation and monitoring of children's health-related quality of life. At the same time, it has the potential to improve intervention programs and relevant and effective planning, optimising their adequacy in each socio-cultural context. Data collected through a reliable instrument to assess health-related quality of life allows monitoring of the health of children, one of the key issues in both public health and health psychology (Ribeiro, 1994). 


\section{ACKNOWLEDGEMENTS}

The authors would like to thank all the Aventura Social team for the collaboration in data collection and analysis (www.aventurasocial.com) and Professor Doctor Ulrike Ravens-Sieberer (KIDSCREEN European Group Coordinator) for methodological assistance and finally all The KIDSCREEN Group Europe (www.KIDSCREEN.org).

Authors gratefully acknowledge the help from the reviewers of the Journal of Family Studies with regard to methodological issues and English language usage.

\section{References}

Bisegger, C., Cloetta, B., Rueden, U., Abel, T., Ravens-Sieberer, \& European KIDSCREEN Group. (2005). Health-related quality of life: Gender differences in childhood and adolescence. Soz-Praventivmed 50, 281-291.

Bronfenbrenner, U. (1986). Ecology of the family as a context for human development: Research perspectives. Developmental Psychology 22, 723-742.

Caldera, Y., \& Hart, S. (2004). Exposure to child care parenting style and attachment security. Infant and Child Development 13, 21-33.

Chang, P., \& Yeh, C. (2005). Agreement between child self-report and parent proxy-report to evaluate quality of life in children with cancer. Psycho Oncology 14, 125-134.

Cohen, J. (1988). Statistical power analysis for the behavioral sciences (2nd ed). Hillsdale NJ: LEA

Currie, C., Samdal, O., Boyce, W., \& Smith, R. (2001). HBSC - a WHO cross national study: Research protocol for the 2001/2002 survey. Copenhagen: WHO

Eiser, C., \& Morse, R. (2001). Can parents rate their child's health-related quality of life? Results of a systematic review. Qual Life Res 10, 347-357.

Gaspar, T., \& Matos, M. (Eds). (2008). Versão portuguesa dos instrumentos KIDSCREEN-52: Instrumentos de Qualidade de Vida para Crianças e Adolescentes (Portuguese version of KIDSCREEN52 Instruments: Quality of life instruments for children and Adolescents). Lisbon: FMH.

Gaspar, T., Matos, M.G., Ribeiro, J., \& Leal, I. (2005). Saúde qualidade de vida e desenvolvimento (Health quality of life and development). In Matos MG (Ed.). Comunicação Gestão de Conflitos e Saúde na Escola (pp.119124). (Communication conflict management and health in school). Lisbon: FMH.
Gaspar, T., Matos, M., Ribeiro, J.L., \& Leal, I. (2006). Qualidade de vida e bem-estar em crianças e adolescentes. Revista Brasileira de Terapias Cognitivas 2(2), 47-60.

Gaspar, T., Ribeiro, J., Matos, M., Leal, I., \& Ferreira, A. (2009a). Psychometric Properties of a Brief Version of the Escala de Satisfação com o Suporte Social for Children and Adolescents. The Spanish Journal of Psychology, 12(1), 360-372

Gaspar, T., Matos, M Ribeiro, J., Leal, I., \& Ferreira, A. (2009b). Health-related quality of life in children and adolescents and associated factors. Journal of Cognitive and Behavioral Psychotherapies, 9(1), 33-48.

Gaspar, T., Matos, M., Ribeiro, J.L., Leal, I., Erhart, M., \& Ravens-Sieberer, U. (2010). Kidscreen: Quality of life in children and adolescents. Journal of Child and Adolescent Psychology, 1, 49-64.

Harding, L. (2001). Children's quality of life assessments: A review of genetic and health related quality of life measures completed by children and adolescents. Clinical Psychology and Psychotherapy 8, 79-96.

Jokovic, A., Locker, D., \& Guyatt, G. (2004). How well do parents know their children? Implications for proxy reporting of children health-related quality of life. Quality of life Research 13, 1297-1307.

Kazdin, A., \& Whitley, M. (2003). Treatment of parental stress to enhance therapeutic change among children referred for aggressive and antisocial behaviour. Journal of Consulting and Clinical Psychology 71(3), 503-515.

Marshall, G., Hays, R., \& Nicholas, R. (1994). Evaluating agreement between clinical assessment methods. Int J Method Psych 37, 249-257.

Matos, M.G. (2005). Comunicação Gestão de Conflitos e Saúde na Escola (Communication conflict management and health in school). Lisboan: FMH.

Matos, M., Simões, C., Tomé, G., Gaspar, T., Camacho, I., Diniz, J., \& Equipa do Aventura Social (2006). A Saúde dos Adolescentes Portugueses - Hoje e em 8 anos - Relatório Preliminar do Estudo HBSC 2006.

Website:www.fmh.utl.pt/aventurasocial; www.aventurasocial.com

Mccullough, G., Huebner, E., \& Laughlin, J. (2000). Life events self-concept and adolescents' positive subjective well-being. Psychology in the Schools 37 (3), 281-290.

Nelson, G., Laurendeau, M., \& Chamber, I.C. (2001). A review of programs to promote family wellness and prevent the maltreatment of children. Canadian Journal of Behavioural Science 33(1), 1-13. 
Plancherel, B., Bolognini, M., \& Halfon, O. (1998). Coping strategies in early and midadolescence: Differences according to age and gender in community sample. European Psychologist 3(3), 192-201.

Ravens-Sieberer, U., Gosch, A., Abel, T., Auquier, P., Bellach, B., Bruil, J., Dur, W., Power, M., Rajmil, L., \& European KIDSCREEN Group. (2001). Quality of life in children and adolescents: A European public health perspective. Preventive Med 46, 294-302.

Ravens-Sieberer, U., Gosch, A., Rajmil, L., Erhart, M., Bruil, J., Duer, W., Auquier, P., Power, M., Abel, T., Czemy, L., Mazur, J., Czimbalmos, A., Tountas, Y., Hagquist, C., Kilroe, J., \& KIDSCREEN group (2005). KIDSCREEN-52 quality-of-life measure for children and adolescents. Expert Rev Pharmacoeconomics Outcomes Res 5(3), 53-61.

Ribeiro, J. L. P. (1994). A Importância da Qualidade de Vida para a Psicologia da Saúde (The importance of Quality of life to Health Psychology). Análise Psicológica 2-3 (12), 179-191.

Rueden, U., Gosch, A., Rajmil, L., Bisegger, C., Ravens-Sieberer, U., \& European KIDSCREEN Group. (2006). Socioeconomic determinants of health related quality of life in childhood and adolescence: Results from a European study. $J$ Epidemiol Community Health 60, 130-135.

Sung, L., Young, N.L. , Greenberg, M.L., McLimont, M., Samanta, T., Wong, J., Rubenstein, J., Ingber, S., Doyle, J.J., \& Feldman, B.M. (2004). Healthrelated quality of life (HRQoL) scores reported from parents and their children with chronic illness differed depending on utility elicitation method. Journal of Clinical Epidemiology 57, 1161-1166.

Tamim, H., McCusker, J., \& Dendukuri, N. (2002). Proxy-reporting of quality of life using the EQ-5D. Med Care 40, 1186-1195.

\title{
NOW A V A I L A B L E
}

\section{Social Determinants of Child Health and Wellbeing}

\author{
Special Issue of Health Sociology Review
}

ISBN: 978-1-921348-15-0 Volume 18 Issue $1 \sim$ ii+134 pages June 2009

Editors: Jianghong Li, Centre for International Health and School of Public Health, Curtin University of Technology; Fiona Stanley and Eugen Mattes, Telethon Institute for Child Health Research, Centre for Child Health Research, University of Western Australia; Anne McMurray, Peel Health Campus Chair in Nursing, Murdoch University; and Clyde Hertzman, Director Human Early Learning Partnership, University of British Columbia

There are growing concerns about the increasingly poor health outcomes for today's children and youth in both developed and developing countries. This special issue addresses the social inequalities connected with child health/well-being, and the social, political, and cultural factors which shape (or determine) child health/well-being.

eContent Management Pty Ltd, PO Box 1027, Maleny QLD 4552, Australia

Tel.: +61-7-5435-2900; Fax. +61-7-5435-2911; subscriptions@e-contentmanagement.com www.e-contentmanagement.com

\section{B R A R Y R C O M M E D A T I O N F O R M}

Free inspection copies of Journal of Family Studies now available for consideration by your faculty's library selection committee. Please photocopy the form below, attach it to this copy of the journal and forward both to the committee representative in your school. We shall send you replacement copies on request - please contact the journal promptly at: subscriptions@e-contentmanagement.com

I recommend that the library subscribes to Journal of Family Studies for the following reasons:

$\square$ I have evaluated the content of this journal and can confirm that the high quality of the articles will be of significant benefit to faculty and students in teaching and research

$\square$ The journal has core content which is central to the research and teaching interests of my department

$\square \mathrm{I}$ am an author / member of the editorial board and as such will be using it regularly for research, article submission and as a teaching aid.

$\square$ I will be using this journal for my student course packs and reading lists

$\square$ I will be using this journal for my own research and scholarship

$\square$ I have noted the journal is frequently cited and is therefore a critical acquisition for our collection in this subject area

Requested by: Faculty/Library: School (if applicable):

Date: Email: Website: 
Copyright of Journal of Family Studies is the property of eContent Management Pty. Ltd. and its content may not be copied or emailed to multiple sites or posted to a listserv without the copyright holder's express written permission. However, users may print, download, or email articles for individual use. 